\title{
Optimization of New Sample Preparation Technique for the Determination of Methadone and Codeine in Plasma Sample by GC-FID
}

\author{
Lazgin A. Jamil ${ }^{\circledR *, a}$ \\ ${ }^{a}$ Department of Chemistry, Faculty of Science, University of Zakho, 42002 Zakho, Iraq
}

\begin{abstract}
A continuous sample drop flow microextraction (CSDF-ME) technique was modified for the pretreatment of plasma samples in the determination of methadone and codeine. The limit of detection and linear range for methadone were $15.0 \mu \mathrm{g} \mathrm{L}^{-1}$ and $0.1-10.0 \mathrm{mg} \mathrm{L}^{-1}$, respectively. The detection limit and linear range for codeine were $25.0 \mu \mathrm{g} \mathrm{L}^{-1}$ and $0.2-15.0 \mathrm{mg} \mathrm{L}^{-1}$, respectively. The extraction recoveries were 41.6-52.1\%, and the enrichment factors ranged from 65 to 80 for codeine and methadone, respectively. Intra- and interday precisions of the technique were calculated for codeine and methadone concentrations of $0.4 \mathrm{mg} \mathrm{L}^{-1}$ and were $4.2-7.6 \%$ and $2.5-5.1 \%$, respectively. Also, the accuracy (error\%) of method was in the range -10 to $+7.5 \%$. The applicability of the CSDF-ME-gas chromatography-flame ionization detector (GC-FID) technique was illustrated by the determination of the mentioned drugs in human plasma. The attained relative recoveries of methadone and codeine in the 90.0-107.5\% range demonstrated the excellent capability of the optimized technique for the microextraction of the mentioned drugs from plasma. The CSDF-ME-GC-FID is a rapid, repeatable and very simple technique that requires a low sample amount.
\end{abstract}

Keywords: methadone, codeine, continuous sample drop flow microextraction, gas chromatography

\section{Introduction}

The quantitative analysis of drug concentrations in biological samples plays a significant role in drug development and discovery. Sample preparation methods are needed for the bioanalysis of drugs. ${ }^{1}$ The synthetic painkiller methadone (MDN) is prescribed to treat addiction on opioids such as heroin. ${ }^{2} \mathrm{MDN}$ is used as a painkiller for acute to chronic pains, lightening the affected individuals' symptoms and its action of reducing addiction on the drugs as a withdrawal agent has raised overdose cases and use in children and adults. MDN causes up to $32 \%$ of opioid analgesics poisoning. ${ }^{3-8}$ Medical research has shown that the required administered methadone dosage should be based on an evaluation performed by a qualified clinician through a recognized clinical process. ${ }^{9,10}$ To achieve optimum treatment, new analytical methods and time-saving sample preparation techniques for the determination of methadone in blood extract samples have been used. ${ }^{11,12}$ The opiate alkaloid codeine (3-methyl morphine; COD) is extracted from poppy flowers and is produced from morphine by a methylation reaction, ${ }^{13}$ which is extensively used as a

*e-mail: lazgin.jamil@uoz.edu.krd cough and pain reliever. ${ }^{14} \mathrm{COD}$ is an inactive drug yet, by the liver enzyme (CYP2D6) can be demethylated to active morphine, which is considered fatal for its excessive intake and can be addictive for long-term use. The drug spreads throughout the body by its absorption from the gastrointestinal tract and then spreading through intravascular gaps to the body tissues with privilege uptake in liver, spleen, and kidney. ${ }^{15,16}$ These two drugs have direct effects on the central nervous system due to their analgesic and anti-depressive properties. ${ }^{17}$ The unmonitored administration of COD and MDN could lead to serious side effects or even death. ${ }^{18}$

The participation of the World Anti-Doping Agency (WADA) with some other related organizations such as International Olympic Committee, Anti-Doping Organizations and International Federations, WADA accredited laboratories, set $50 \mu \mathrm{g} \mathrm{L}{ }^{-1}$ level in the interdicted list and minimum required performance limits (MRPLs) for narcotic drugs. ${ }^{19,20}$ These drugs can offer an increasing analgesic effect and fast pain relief but can also exhibit severe side effects. ${ }^{21-23}$ Their unmonitored or aberrant use can lead to critical medical health complications, and their overdose may be harmful to organs or can even lead to death..$^{15}$ Due to the worldwide usage of these drugs and to 
avoid unfavorable effects and to determine poisoning, it is important to develop suitable analytical methods. These should be less time-consuming than the extraction methods from human fluids with high recovery yields and accurate analytical methods. ${ }^{14,24}$

Various sample preparation techniques have been developed in previous work for the extraction and concentration of methadone and codeine in distinct real samples. These methods include solid-phase extraction (SPE), ${ }^{1}$ dispersive solid-phase microextraction (DSPME),${ }^{25}$ dispersive liquid-liquid microextraction (DLLME), ${ }^{11,19}$ air-assisted emulsification liquid-liquid microextraction (DES-AAELLME) ${ }^{12}$ ultrasound-assisted dispersive liquidliquid microextraction (UA-DLLME), ${ }^{26}$ homogenous liquid-liquid microextraction (HLLME), ${ }^{27}$ hollow fiber liquid-phase microextraction (HF-LPME) ${ }^{28}$ solvent bar microextraction $(\mathrm{SBME})^{29}$ and electromembrane extraction (EME).$^{30}$ The mention methodologies have been utilized for the determination of MDN and COD in various biological matrices, such as urine, ${ }^{11,12,18,28-30}$ plasma or blood, ${ }^{11,12,19,28-30}$ saliva ${ }^{11}$ and sweat. ${ }^{11}$ SPE-based sample preparation delivers enrichment of analytes at trace levels and improvement of sample cleanup. However, it incorporates multiple steps such as the washing and evaporation of the solvents, requires higher costs per sample with usage of large sample volumes. ${ }^{31-34}$ SPME have significant advantages that merges sampling, microextraction, preconcentration and sample introduction into one step. ${ }^{35,36}$ The process of desorption and the nature of polymeric extraction phase have enormous relation in SPME method drawbacks; in fact, low repeatability and batch-to-batch variation are the results of the utilization of polymer as an extraction phase..$^{29,37}$ The DLLME is defined for its simplicity, high speed of extraction, ease of operation, high extraction recovery with high enrichment factor and simple equipment requirement. ${ }^{38-40}$ Moreover, the existed particles in the biological samples during sample/acceptor phase separation can sediment at the bottom of the DLLME used device or suspend on the surface of its filled solution. HLPME comparing to DLLME delivers higher extraction efficiency; however, the selectivity of the HLPME method tends to be reduced which is not appropriate for the complex matrices, especially biological samples. In addition, HLPME tends to use larger volumes of organic solvents causing in difficulty of the collection of the extraction phase in complex matrices. ${ }^{39,41}$ The HF-LPME is presenting substantial sample cleanup comparing with the other LPME methods, making it a reasonable method to be widely used for the analysis of pharmaceuticals from biological samples. ${ }^{39}$ Long extraction time is considered the main disadvantage of the conventional HF-LPME due to the passive diffusion, ${ }^{42}$ which was remarkably decreased with the introduction of EME. ${ }^{39}$ The main drawback of EME is more problematic when samples with high ionic contents, high voltages or polar organic solvents such as plasma and urine are employed..$^{39}$ During extraction, increasing the sample agitation improves SBME extraction efficiency ${ }^{43}$ In SBME, the transfer of analytes highly increases from the aqueous sample to the extraction solvent by the free movement of the solvent bar in an aqueous sample solution. ${ }^{29,43}$ However, reusing the membrane forms memory effect and requirement for the preconditioning of the membrane results in a limitation of the SBME method. ${ }^{39}$

To overcome the highlighted difficulties, Moinfar et al. ${ }^{44}$ developed a new microextraction technique named as continuous sample drop flow microextraction (CSDF-ME).

In CSDF-ME, the conical bottom of the extraction tube is filled with a high-density water-immiscible organic solvent, and afterwards the aqueous solution is pumped through the extraction solvent. In this process, the analyte of interest are extracted from the droplets by the organic solvent phase at the bottom of tube and preconcentrated there. The organic solvent phase is used for analysis by a micro syringe. Only small amounts of the extraction solvent are needed for the CSDF-ME method. Using this extraction method, a high enrichment factor can be obtained, and the method is easy to operate. This method has also been proven to be reproducible and more efficient than other LPME methods. This is due to the decrease in the stages of the sample preparation methods. The efficient extraction of benzene, toluene, ethylbenzene and xylenes (BTEX) ${ }^{44}$ chlorophenols ${ }^{45}$ triazine herbicides ${ }^{46}$ and heavy metal compounds ${ }^{47-50}$ using the CSDF-ME method has been demonstrated. For the first time, CSDF-ME was developed for determination and pre-concentration of codeine (COD) and methadone (MDN) prior to their analysis by gas chromatography-flame ionization detector (GC-FID), which presented simplicity and specificity for obtaining the determination of COD and MDN in plasma sample. The results obtained using this method demonstrate that CSDFME is a suitable and efficient microextraction technique for the extraction and analysis of methadone and codeine in a plasma matrix.

\section{Experimental}

\section{Chemicals and reagents}

Codeine and methadone were supplied by SigmaAldrich (St. Louis, MO, USA). $1000 \mathrm{mg} \mathrm{L}^{-1}$ of these drugs were prepared as stock standard solution in methanol (SupraSolv ${ }^{\circledR}$, Merck, Darmstadt, Germany) and kept at 
$-20{ }^{\circ} \mathrm{C}$. Working solutions $\left(0.5 \mathrm{mg} \mathrm{L}^{-1}\right)$ in ultrapure water were prepared. Chloroform (suprasolvent GC grade), carbon disulfide (GR, grade) and carbon tetrachloride (GR grade) were obtained from Merck (Darmstadt, Germany). For the preparation and dilution of working solution, deionized water $(18 \mathrm{M} \Omega \mathrm{cm})$ was used. Sodium hydroxide pellets and $\mathrm{HCl}$ (65\% solution) were supplied by Merck (Darmstadt, Germany).

Instruments

A Shimadzu gas chromatography (GC 2010, Shimadzu, Japan) with a flame ionization detector and an injector (programmed spilt/splitless system) was performed for the qualitative and quantitative measurements of codeine and methadone. Carrier gas (Ultrapure He, Air Products, UK, 99.9999\%) with a molecular sieve trap was used at a flow rate of $1.0 \mathrm{~mL} \mathrm{~min}^{-1}$. The splitless mode for $0.5 \mathrm{~min}$ was used, and the injector oven was kept at $270{ }^{\circ} \mathrm{C}$. A BP-5, $30 \mathrm{~m} \times 0.25 \mathrm{~mm} \times 0.25 \mu \mathrm{m}$ capillary column (Phenomenex, USA) was used. The oven temperature program was adjusted as follows: initial $100{ }^{\circ} \mathrm{C}(2 \mathrm{~min})$ to $300{ }^{\circ} \mathrm{C}$ at a rate of $40{ }^{\circ} \mathrm{C} \min ^{-1}(7 \mathrm{~min})$. The FID was kept at $300{ }^{\circ} \mathrm{C}$, and a Shimadzu hydrogen generator (OPGU-2200s) with a $40 \mathrm{~mL} \mathrm{~min}^{-1}$ flow rate was used for FID fuel. The oxygen (99.999\%, Air Products) flow rate of $400 \mathrm{~mL} \mathrm{~min}{ }^{-1}$ was applied for FID. The $1.0 \mu \mathrm{L}$ of extraction solvent volume was injected to GC for all the samples.

A Metrohm pH-meter (model 691) was used. A Heidolph peristaltic pump, (PD5001, UK) provided the supply at several flow rates.

\section{Microextraction procedure}

Sample solution or pure water $(2.5 \mathrm{~mL})$ with a $\mathrm{pH}$ of 10 was spiked at the level of $0.5 \mathrm{mg} \mathrm{L}^{-1}$ with methadone and codeine. Chloroform $(25.0 \mu \mathrm{L})$ (extraction solvent) was placed in a small conical bottom tube. The narrow needle was placed inside the extraction solvent, and then the sample solution $(2.5 \mathrm{~mL})$ was passed through the organic solvent at a flow rate of $0.5 \mathrm{~mL} \mathrm{~min}{ }^{-1}$ by a peristaltic pump. The sample droplets were sequentially introduced into the organic solvent. The drugs were extracted from the sample droplets into the organic solvent. At the end of extraction, the organic solvent remained at the bottom of the small tube. CSDF-ME method is shown in Figure 1.

An aliquot $(1.0 \mu \mathrm{L})$ of the chloroform extract was removed by a micro syringe (SGE) and introduced into the GC. The remaining chloroform volume was measured by a $25 \mu \mathrm{L}$ micro syringe and was found to be $16 \pm 0.3 \mu \mathrm{L}$.

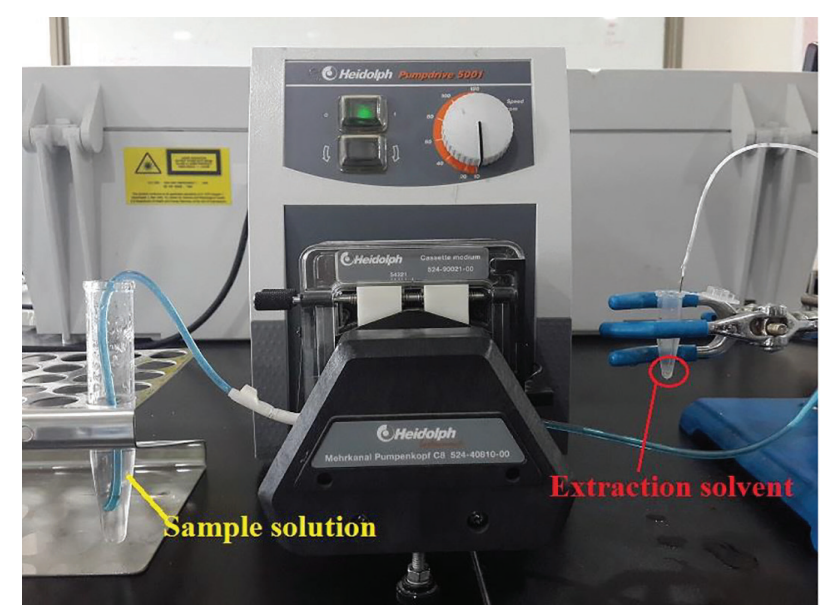

Figure 1. Continuous sample drop flow microextraction procedure.

\section{Sample preparation}

The plasma sample $(1.0 \mathrm{~mL})$ was transferred to a glass tube, and a $0.5 \mathrm{~mL}$ of $15 \%(\mathrm{~m} / \mathrm{v}) \mathrm{ZnSO}_{4}$ solution and $0.3 \mathrm{~mL}$ of acetonitrile was added. The solution was shaken for $5 \mathrm{~min}$ and stored for $4 \mathrm{~min}$ at $5{ }^{\circ} \mathrm{C}$ and then centrifuged at $5000 \mathrm{rpm}$ ( $5 \mathrm{~min}$ ). The supernatant solution was diluted in another glass tube with pure water to $2.5 \mathrm{~mL}$. Then, the solutions $(2.5 \mathrm{~mL})$ were removed for analysis as demonstrated in the sub-section "Microextraction procedure". Plasma sample was obtained from the Clinic of Beidar Hospital (Zakho, Iraq) and a plasma sample which content of drugs was obtained from a volunteer who had been treated with methadone and codeine. This study was approved by the Research Ethics Committee of Clinic of Beidar Hospital (Zakho, Iraq) under No. 018254.

\section{Calculation of recovery and enrichment factor}

In this research, for the first time, codeine and methadone were chosen as examples for study by CSDF-ME combined with GC-FID. Different factors were optimized to achieve a high extraction recovery (ER) and enrichment factor (EF). In the CSDF-ME method, many factors, such as the $\mathrm{pH}$, extraction solvent volume and type, and aqueous solution flow rate and volume, can affect the microextraction of codeine and methadone.

$\mathrm{EF}$ is defined as the ratio of the drug concentration in the remaining organic solvent $\left(\mathrm{C}_{\mathrm{rem}}\right)$ to the concentration of the drug in the initial solution $\left(\mathrm{C}_{0}\right)$ :

$\mathrm{EF}=\frac{\mathrm{C}_{\mathrm{rem}}}{\mathrm{C}_{0}}$

ER is defined by the total ratio of the amount of the extracted drug in the remaining organic phase $\left(\mathrm{n}_{\text {rem }}\right)$ to 
the amount of the drug in the initial aqueous solution $\left(\mathrm{n}_{0}\right)$. Additionally, $\mathrm{C}_{\mathrm{rem}}$ is the concentration of the drug in the remaining organic phase, which is obtained from an external standard calibration curve of the drug's standard solution.

$\operatorname{ER}(\%)=\frac{\mathrm{n}_{\mathrm{rem}}}{\mathrm{n}_{0}} \times 100=\frac{\mathrm{C}_{\mathrm{rem}} \times \mathrm{V}_{\mathrm{rem}}}{\mathrm{C}_{0} \times \mathrm{V}_{\mathrm{aq}}} \times 100$

$\mathrm{ER}(\%)=\left(\mathrm{V}_{\mathrm{rem}} / \mathrm{V}_{\mathrm{aq}}\right) \mathrm{EF} \times 100$

where $\mathrm{V}_{\mathrm{aq}}$ and $\mathrm{V}_{\mathrm{rem}}$ are the aqueous sample and remaining organic solvent volume, respectively. Additionally, the relative recovery (RR\%) of the spiked samples was obtained according to:

$\mathrm{RR}(\%)=\frac{\mathrm{C}_{\text {found }}-\mathrm{C}_{\text {real }}}{\mathrm{C}_{\text {added }}} \times 100$

where $\mathrm{C}_{\text {found }}, \mathrm{C}_{\text {real }}$, and $\mathrm{C}_{\text {added }}$ are the total concentration of the drug after the addition of the standards in the plasma, the original concentration of the drug prior to the addition of the standard in the plasma and the concentration of the drug added to the plasma, respectively.

\section{Results and Discussion}

$\mathrm{pH}$ selection

The $\mathrm{pH}$ of the aqueous solution influences the neutral or ionic forms of codeine and methadone. The solubility of the drugs in aqueous solutions and their affinity toward the organic solvent is strongly affected by the changes in the $\mathrm{pH}$. For the study of CSDF-ME, different tests were carried out at various $\mathrm{pH}$ levels of the aqueous sample in the 5.0-12.0 range. The results shown in Figure 2 indicated that by increasing $\mathrm{pH}$ from 5.0 to 8.0 , the EFs of codeine and methadone are improved due to their basic nature with $\mathrm{p} K_{\mathrm{a}}$ 10.6 and 8.94. As the $\mathrm{pH}$ increases these drugs change to their nature form with decrease in their solubility in water, causing the improvement of codeine and methadone extraction. As illustrated in Figure 2, by increasing the $\mathrm{pH}$ of the sample solution the EF maximizes until $\mathrm{pH} 10$ while at higher $\mathrm{pH}$ values the EF slightly degrades due to the alkalinity of the solution in which the interferences compete with the target analytes in the biological samples to extraction solvent. Therefore, the $\mathrm{pH} 10.0$ was selected for the sample solution.

Investigation of type and volume of the extraction solvent

In CSDF-ME, the extraction solvent must be water-

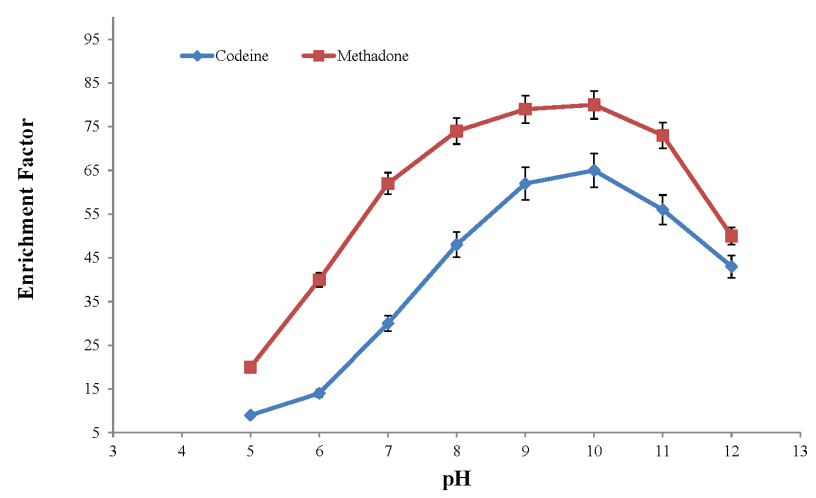

Figure 2. Effect of $\mathrm{pH}$ on the enrichment factors of codeine and methadone achieved from CSDF-ME method. Extraction conditions: chloroform volume, $25.0 \mu \mathrm{L}$; aqueous sample volume, $2.5 \mathrm{~mL}$; drug concentration, $0.4 \mathrm{mg} \mathrm{L}^{-1}$; flow rate of sample, $0.5 \mathrm{~mL} \mathrm{~min}^{-1}$.

immiscible and have a higher density than water. In the solubility tests of codeine and methadone, several extraction solvents, namely, carbon tetrachloride, chloroform and carbon disulfide, were examined. The compatibility of the organic solvents with the CSDF-ME method was investigated using an aqueous solution $(2.5 \mathrm{~mL})$ containing codeine and methadone with $\mathrm{pH} 10.0$. Since different solubility's are obtained for the organic solvents in an aqueous solution. To achieve constant volume of the remaining phase for all of the organic solvents $(16.0 \mu \mathrm{L})$, a series of tests with various volumes of extraction solvent were performed using carbon tetrachloride $(19.3 \mu \mathrm{L})$, carbon disulfide $(21.5 \mu \mathrm{L})$ and chloroform $(25.0 \mu \mathrm{L})$. Extraction recovery was calculated for all three solvents and the maximum extraction recovery was obtained when chloroform (41.0 and $52.0 \%$ for codeine and methadone, respectively) was applied compared to two other solvents. That is most probably due to the higher polarity of the chloroform giving it the advantage over the other two solvents to extract MDN and COD better. Therefore, chloroform was selected as optimal extraction solvent.

The next factor to be considered is the organic solvent volume. According to the previously reported study, ${ }^{44}$ the organic solvent volume should be kept to a minimum in order to attain the highest possible EF and to prevent pollution of the environment. However, in the study of the effect of solvent volumes, the volume cannot be set lower than $25 \mu \mathrm{L}$, due to the substantial reduction of the contact between sample droplets and extraction phase as the solvent level in the extraction vessel is reduced. Consequently, the sample flow has a great influence on the volume of solvent and remained solvent stability. Therefore, in volumes below $25 \mu \mathrm{L}$, repeatability decreases sharply.

The effects of the organic solvent volume were investigated by various volumes of chloroform $(25.0,30.0$, 
35.0 and $45.0 \mu \mathrm{L}$ ) with the CSDF-ME method. Figure 3 shows the changes in EF plotted versus the extraction solvent volume. It is observed that the enrichment factor decreases with increasing chloroform volume due to enhancement in the remaining chloroform volume. Consequently, the highest enrichment factor was obtained with $25.0 \mu \mathrm{L}$ chloroform. Therefore, $25.0 \mu \mathrm{L}$ was chosen as the optimal volume of chloroform. In many microextraction techniques, due to the gain in the sensitivity, the enrichment factor is more important than ER, and researchers attempt to keep it as high as possible. ${ }^{49}$

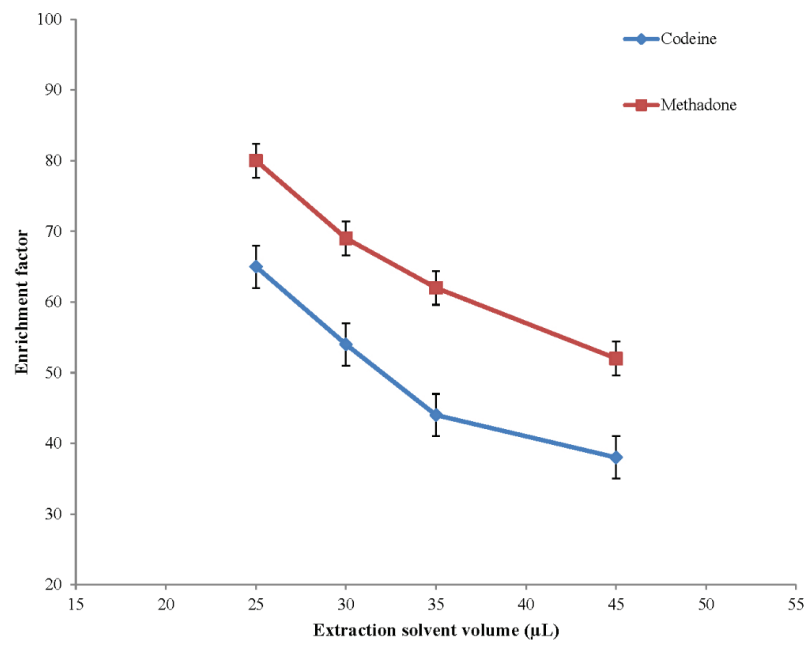

Figure 3. Effect of the chloroform volume (extraction solvent) on the enrichment factors of codeine and methadone achieved from CSDF-ME. Drug concentration, $0.4 \mathrm{mg} \mathrm{L}^{-1}$; volume of sample, $2.5 \mathrm{~mL}$; $\mathrm{pH} 10.0$; flow rate of sample solution, $0.5 \mathrm{~mL} \mathrm{~min}^{-1}$.

Flow rate of the aqueous solution

According to the previously reported studies ${ }^{44-50}$ of CSDF-ME method to reach the highest EF, the flow rate of the sample solution is kept at its minimum. However, for the interests of saving procedure time flow rates lower than $0.5 \mathrm{~mL} \mathrm{~min}^{-1}$ were not considered. In the CSDF-ME method, as increase in the flow rate of aqueous solution causes decrease in contact time between the sample droplets and the extraction solvent decreases. The influence of the aqueous solution flow rate in the range of $0.5-2.0 \mathrm{~mL} \mathrm{~min}^{-1}$ was investigated. As the results are showed in Figure 4, by increasing the flow rate of water samples the EF decrease sharply, due to the shorter contact time of sample droplets with extraction phase as the speed of pumping the sample raises to the solvent resulting in decreases extraction of $\mathrm{MDN}$ and COD by the extraction phase. At flow rates above $1.0 \mathrm{~mL} \mathrm{~min}{ }^{-1}$, the droplet does not form, and the sample quickly passes through the extraction solvent; thus, the enrichment factor sharply decreases. Hence, $0.5 \mathrm{~mL} \mathrm{~min}^{-1}$ was chosen as the optimal flow rate for the subsequent extractions.

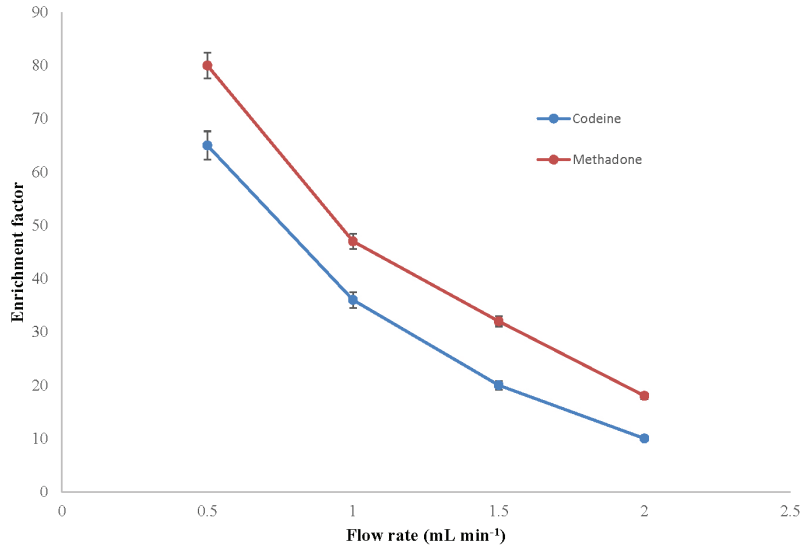

Figure 4. Effect of the sample solution flow rate on the enrichment factor of codeine and methadone attained from CSDF-ME. Extraction conditions: drug concentration, $0.4 \mathrm{mg} \mathrm{L}^{-1}$; volume of aqueous sample, $2.5 \mathrm{~mL}$; $\mathrm{pH}$ 10.0; chloroform volume, $25.0 \mu \mathrm{L}$.

\section{Selection of the aqueous sample volume}

The influence of the aqueous solution volume in the range of 2.5-4.0 mL on EF was evaluated. The different volumes of sample solutions were pumped into the extraction solvent under constant conditions. As illustrated in Figure 5, the enrichment factor increased with increasing sample volume from 2.5 to $4.0 \mathrm{~mL}$, due to the increases of the amount of dissolved drugs (in the fixed concentration) and therefore amount of extracted drugs increases in the extraction solvent. However, by increasing of sample volume, the time required for sample pumping across the organic solvent is increased from 5.0 to $8.0 \mathrm{~min}$ at a fixed flow rate $\left(0.5 \mathrm{~mL} \mathrm{~min}^{-1}\right)$. Therefore, a volume of $2.5 \mathrm{~mL}$ was selected to save the extraction time as well as the sample volume consumption.

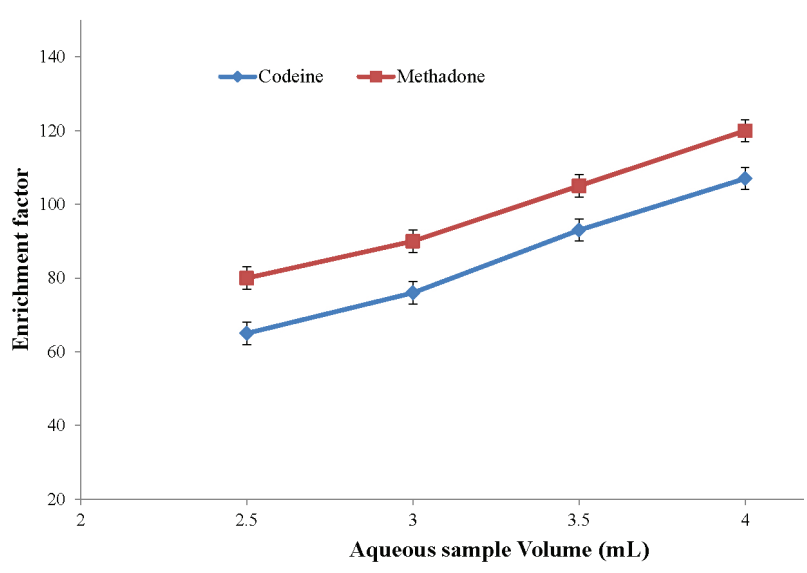

Figure 5. Effect of the aqueous solution volume on the enrichment factors of codeine and methadone from CSDF-ME. Extraction conditions: drug concentration, $0.4 \mathrm{mg} \mathrm{L}^{-1}$; chloroform volume, $25.0 \mu \mathrm{L} ; \mathrm{pH} 10.0$; flow

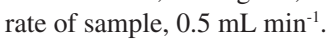

All of the factors have been optimized and microextraction have been performed under optimal 
conditions for real plasma samples, which included; extraction of methadone and codeine into $25.0 \mu \mathrm{L}$ of chloroform was carried out from $2.5 \mathrm{~mL}$ of the plasma samples with $\mathrm{pH} 10.0$ at flow rate of $0.5 \mathrm{~mL} \mathrm{~min}^{-1}$.

\section{Method validation}

The applicability of the combination of CSDF-ME technique with GC-FID for the quantitative determination of methadone and codeine in plasma was examined. The present technique was validated in terms of analytical performance, including precision (intraday and interday), extraction recovery (ER), limit of detection (LOD), linear range (LR) and enrichment factor (EF). The figures of merits of the technique are summarized in Table 1. The reproducibility (interday) and repeatability (intraday) of the technique were investigated by extraction of drugs from the spiked plasma ( $0.4 \mathrm{mg} \mathrm{L}^{-1}$ for each drug). The relative standard deviations (RSDs) (intraday) were measured with five determinations of the drugs within one day, while the RSD (interday) was examined by the determination of drugs in the spiked samples for five successive days. Interday and intraday precisions of the technique were acceptable relative to the standard deviations (RSDs) for codeine (7.6 and 4.2\%) and methadone (5.1 and 2.5\%). Linearity of calibration curves were obtained in the range of $0.2-15$ and $0.1-10 \mathrm{mg} \mathrm{L}^{-1}$ for codeine and methadone, respectively. The ranges of square correlation coefficients $\left(\mathrm{r}^{2}\right)$ were found to vary from 0.999 to 0.998 . The fine detection limits, based on a signal-to-noise ratio (S/N) of 3, were 25.0 and $15.0 \mu \mathrm{g} \mathrm{L}^{-1}$ for codeine and methadone, respectively. The extraction recoveries and enrichment factors of this method for codeine and methadone were in the ranges of $41.6-52.1 \%$ and 65-80, respectively.

\section{Application of real sample}

To study the accuracy and applicability of the present microextraction for the extraction and determination of codeine and methadone, a plasma sample was analyzed by GC-FID after the CSDF-ME method was used. The results showed that codeine and methadone were not found in the plasma sample. To assess the matrix effects, a real plasma sample at two concentration levels was spiked with codeine and methadone standards. The relative recovery of the plasma sample results are summarized in Table 2. As observed from the results, the RRs\% for codeine and methadone in the spiked plasma were found to vary from 90.0 to $102.0 \%$. The error percentages (E\%) as accuracy of the technique for the codeine and methadone were obtained in the range of -0.1 to $-1.6 \%$ in plasma which indicated the

Table 1. Figure of merit data for CSDF-ME and GC-FID

\begin{tabular}{|c|c|c|c|c|c|c|c|c|c|c|c|}
\hline \multirow{2}{*}{ Drug } & \multirow{2}{*}{$\begin{array}{l}\mathrm{LOD}^{\mathrm{b}} / \\
\left(\mu \mathrm{g} \mathrm{L} \mathrm{L}^{-1}\right)\end{array}$} & \multirow{2}{*}{$\mathrm{EF}^{\mathrm{c}}$} & \multirow{2}{*}{$\mathrm{ER}^{\mathrm{d}} / \%$} & \multicolumn{3}{|c|}{$\mathrm{RSD}^{\mathrm{e}} / \%$ (intra-day) } & \multicolumn{3}{|c|}{$\mathrm{RSD}^{\mathrm{e}} / \%$ (inter-day) } & \multirow{2}{*}{$\begin{array}{c}\mathrm{LR}^{\mathrm{f}} / \\
\left(\mathrm{mg} \mathrm{L}^{-1}\right)\end{array}$} & \multirow{2}{*}{$\mathrm{r}^{2}$} \\
\hline & & & & 0.2 & 0.4 & 10.0 & 0.2 & 0.4 & 10.0 & & \\
\hline Codeine & 25.0 & 65 & 41.6 & 6.4 & 4.2 & 3.6 & 11.6 & 7.6 & 5.9 & $0.2-15.0$ & 0.998 \\
\hline Methadone & 15.0 & 80 & 52.1 & 4.7 & 2.5 & 2.3 & 8.3 & 5.1 & 4.5 & $0.1-10.0$ & 0.999 \\
\hline
\end{tabular}

a Method validation conditions: chloroform volume, $25.0 \mu \mathrm{L}$; sample volume, $2.5 \mathrm{~mL}$; drugs concentrations, $0.4 \mathrm{mg} \mathrm{L}^{-1}$; flow rate of sample solution,

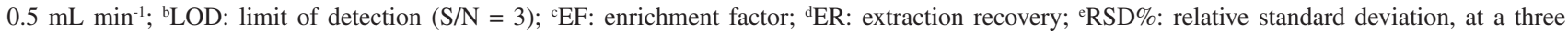
concentration, $0.2,0.4$ and $10.0 \mathrm{mg} \mathrm{L}^{-1}$ of each drug $(\mathrm{n}=5)$; $\mathrm{fR}$ : linear range.

Table 2. Analysis results for determination of spiked real sample ${ }^{\mathrm{a}}$

\begin{tabular}{|c|c|c|c|c|c|}
\hline Sample & Compound & $\mathrm{C}_{\text {added }} /\left(\mathrm{mg} \mathrm{L}^{-1}\right)$ & $\begin{array}{c}\mathrm{C}_{\text {Found }} \\
(\mathrm{SD}, \mathrm{n}=3)^{\mathrm{b}} /\left(\mathrm{mg} \mathrm{L}^{-1}\right)\end{array}$ & Relative recovery / \% & $\mathrm{E} / \%$ \\
\hline \multirow[t]{3}{*}{ Plasma 1} & & 0.0 & 0.0 & - & - \\
\hline & & 0.4 & $0.36 \pm 0.02$ & 90.0 & -10.0 \\
\hline & codeine & 1.0 & $0.97 \pm 0.04$ & 97.0 & -3.0 \\
\hline \multirow[t]{2}{*}{ Plasma 2} & & 0.0 & 0.28 & - & - \\
\hline & & 0.4 & 0.71 & 107.5 & +7.5 \\
\hline \multirow[t]{3}{*}{ Plasma 1} & & 0.0 & not detected & - & - \\
\hline & & 0.4 & $0.39 \pm 0.01$ & 97.5 & -2.5 \\
\hline & methadone & 1.0 & $1.02 \pm 0.03$ & 102.0 & +2.0 \\
\hline \multirow[t]{2}{*}{ Plasma 2} & & 0.0 & 0.22 & - & - \\
\hline & & 0.4 & 0.60 & 95.0 & -5.0 \\
\hline
\end{tabular}

${ }^{a}$ Analysis of real sample conditions: chloroform volume, $25.0 \mu \mathrm{L}$; sample volume, $2.5 \mathrm{~mL}$; flow rate of aqueous sample, $0.5 \mathrm{~mL}$ min ${ }^{-1}$; ${ }^{\mathrm{b}} \mathrm{SD}$ : $\mathrm{standard}$ deviation. $\mathrm{C}_{\text {added }}$ : concentration added; $\mathrm{C}_{\mathrm{Found}}$ : concentration found; E: error percentages. 
presented method is acceptable even in plasma matrices. The chromatograms of the non-spiked plasma sample and the samples spiked with $0.4 \mathrm{mg} \mathrm{L}^{-1}$ of codeine and methadone are shown in Figure 6. In Figure 6a, the small peak is related to methadone, which is less than the linear range of the method.

\section{Comparison of CSDF-ME and other LPME technique}

The determination of codeine and methadone in real sample were compared between optimized CSDF-ME combined with GC-FID and other LPME technique. The results are summarized in Table 3 . The CSDF-ME procedure
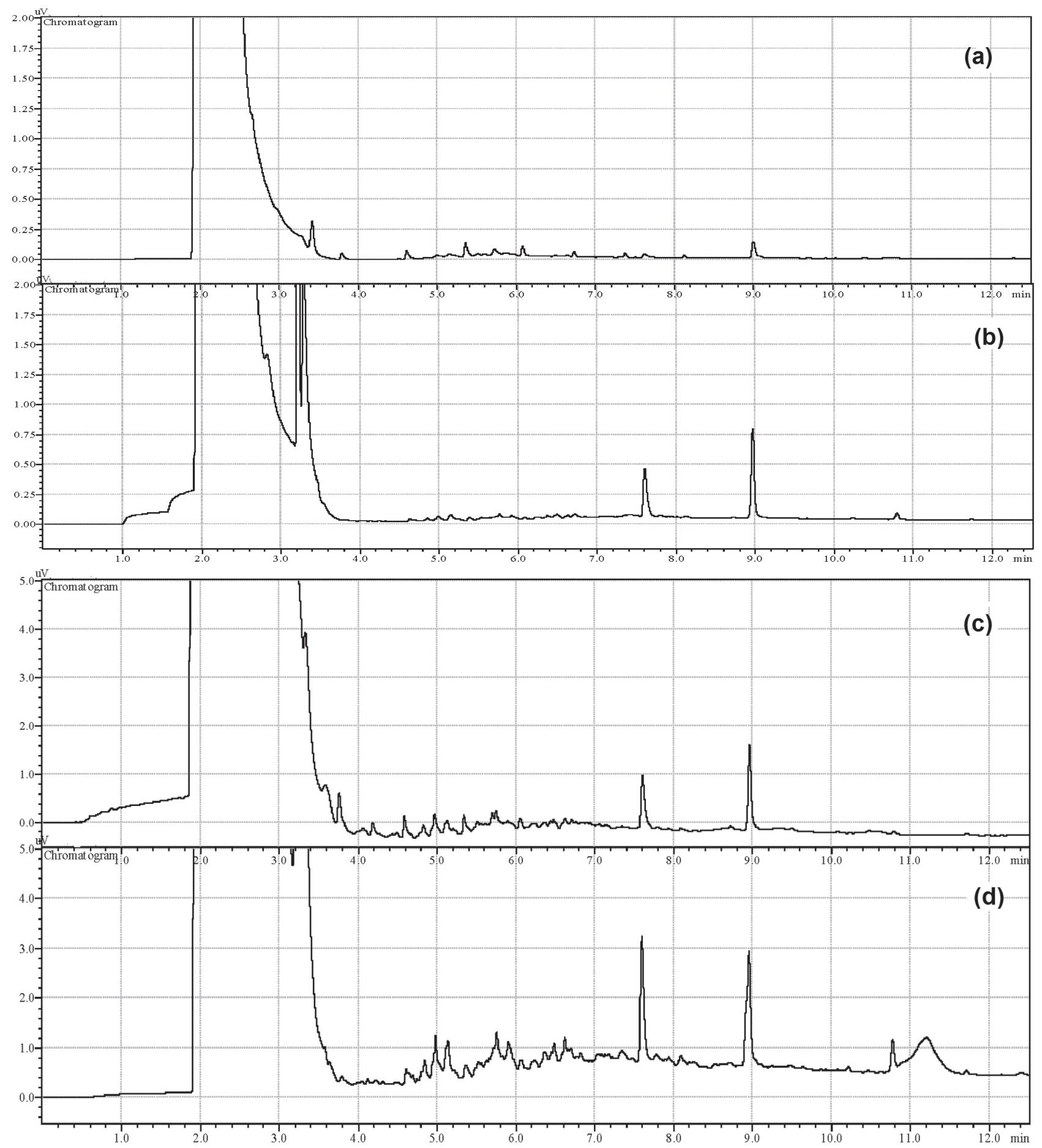

Figure 6. Chromatograms of (a) real plasma sample; (b) spiked real plasma sample from case 1 and (c) real plasma sample containing drugs; (d) spiked real plasma sample from case 2 (spiked: $0.4 \mathrm{mg} \mathrm{L}^{-1}$ of each drug) achieved using CSDF-ME-GC-FID. (1) Codeine; (2) methadone. 
Table 3. Comparison between CSDF-ME technique with other LPME technique for determination of codeine and methadone

\begin{tabular}{|c|c|c|c|c|c|c|c|}
\hline Analyte & Method & $\left.\mathrm{LOD} /(\mu \mathrm{g} \mathrm{L})^{-1}\right)$ & $\mathrm{RSD} / \%$ & Sample / mL & $\begin{array}{l}\text { Extraction } \\
\text { time / min }\end{array}$ & $\mathrm{EF}$ & Reference \\
\hline Methadone & DES-AAELLME-GC-FID ${ }^{\mathrm{a}}$ & 0.7 & 5.4 & 10 & 4 & 270 & 12 \\
\hline Methadone & SBME-GC-FID ${ }^{\mathrm{b}}$ & 7.0 & 7.3 & 10 & 45 & 275 & 29 \\
\hline Codeine & On-chip PEME-HPLC-UV & 5.0 & 2.5 & 2 & - & - & 30 \\
\hline Methadone & SA-PEME-GC-FID ${ }^{\mathrm{d}}$ & 0.6 & 12.7 & 3 & 20 & - & 51 \\
\hline Codeine & EME-HPLC-UV ${ }^{e}$ & 10.0 & 9.5 & 24 & 15 & - & 52 \\
\hline Methadone & UA-DLLME-GC-MS ${ }^{\mathrm{f}}$ & 2.5 & $4.1-6.1$ & 0.2 & 8 & 21 & 26 \\
\hline Codeine & CSDF-ME-GC-FID ${ }^{g}$ & 25.0 & 4.2 & 1 & 5 & 65 & this work \\
\hline Methadone & CSDF-ME-GC-FID ${ }^{g}$ & 15.0 & 2.5 & 1 & 5 & 80 & this work \\
\hline
\end{tabular}

aDeep eutectic solvent air assisted emulsification liquid-liquid microextraction gas chromatography-flame ionization detector; ${ }^{\mathrm{b}}$ solvent bar microextraction gas chromatography-flame ionization detector; ${ }^{\mathrm{c}}$ on-chip pulsed electromembrane extraction high performance liquid chromatography ultravisible detector; ${ }^{\mathrm{d}}$ surfactant assisted pulsed two-phase electromembrane extraction gas chromatography-flame ionization detector; ${ }^{e}$ electromembrane extraction high performance liquid chromatography ultravisible detector; ' ${ }^{\mathrm{f}}$ ultrasound-assisted dispersive liquid-liquid microextraction; ${ }^{\mathrm{g}} \mathrm{continuous} \mathrm{sample} \mathrm{drop} \mathrm{flow}$ microextraction gas chromatography-flame ionization detector. LOD: limit of detection; RSD: relative standard deviation; EF: enrichment factor.

requires less organic solvent with an acceptable recovery. As observed from an examination of the data presented in Table 3, the RSD\% achieved by this technique is lower than those obtained using the other methods. Additionally, the present technique is green compared with the other LPME methods such as DLLME due to the elimination of dispersive solvents. Although the detection limit of the proposed method is relatively higher than other methods, as can be seen from Table 3, the sample volume of the CSDF$\mathrm{ME}$ is less than other methods, that is more appropriate for low volume biological samples. Also, the extraction time of presented method is less than other methods. All of these results indicated that the CSDF-ME-GC-FID technique is a repeatable, sensitive method with simple operation that can be favorably applied for the preconcentration and determination of codeine and methadone.

\section{Conclusions}

The application of the CSDF-ME for the microextraction and determination of codeine and methadone from blood plasma was optimized. The results of present work showed that this technique gives acceptable RR\% and reproducibility for extracting codeine and methadone from plasma samples. A comparison with other methods reported in the literature showed that the present microextraction method has many advantages such as high EF, simplicity, low LOD, low consumption of fewer organic solvents, feasibility of operation, and comparatively short extraction time. Furthermore, the CSDF-ME method appears to have great potential of utilization for the determination of other drugs in biological matrixes as a useful technique for estimating the drug dose effects in patients in clinical trials.
Additionally, the CSDF-ME technique can be optimized for the determination of all analytes that are extractable by suitable organic solvents. But, in this method, the only solvents that are possible to be used are the ones with a higher density than water, which are toxic and may not be suitable for the extraction of some drugs. Thereby, selection for an appropriate solvent has some limitations for fulfilling all the requirements. And also this method has lower EF and LOD than some other LPME methods such as DLLME. It will be useful to test the CSDF-ME method in future studies using organic solvents with lower density than water.

\section{Acknowledgments}

The author is gratefully acknowledged from the University of Zakho for financial support of this research (grant No. UOZ-CH-018-05).

\section{References}

1. El-Beqqali, A.; Abdel-Rehim, M.; Anal. Chim. Acta 2016, 936, 116.

2. Dole, V. P.; Nyswander, M. E.; JAMA, J. Am. Med. Assoc. 1976, 235, 2117.

3. Taheri, S.; Jalali, F.; Fattahi, N.; Jalili, R.; Bahrami, G.; J. Sep. Sci. 2015, 38, 3545.

4. Martin, T. J.; Kahn, W. R.; Xiao, R.; Childers, S. R.; Synapses 2007, 61, 176.

5. Bernard, J.-P.; Opdal, M. S.; Karinen, R.; Mørland, J.; Khiabani, H. Z.; Eur. J. Clin. Pharmacol. 2007, 63, 777.

6. Couper, F. J.; Chopra, K.; Pierre-Louis, M. L. Y.; Forensic Sci. Int. 2005, 153, 71 . 
7. Gharavi, R.; Hedrich, W.; Wang, H.; Hassan, H. E.; Pharm. Res. 2015, 32, 2477.

8. Nakhla, D. S.; Hussein, L. A.; Magdy, N.; Abdallah, I. A.; Hassana, H. E.; J. Chromatogr. B 2017, 1048, 19.

9. Ball, J. C.; Ross, A.; The Effectiveness of Methadone Maintenance Treatment, $1^{\text {st }}$ ed.; Springer-Verlag: New York, USA, 1991.

10. Dole, V. P.; JAMA, J. Am. Med. Assoc. 1988, 260, 3025.

11. Ranjbari, E.; Golbabanezhad-Azizi, A.; Hadjmohammadi, M.; Talanta 2012, 94, 116.

12. Lamei, N.; Ezoddin, M.; Abdi, K.; Talanta 2016, 165, 176.

13. Thorn, C. F.; Klein, T. E.; Altman, R. B.; Pharmacogenet. Genomics 2009, 19, 556.

14. Mohamed, M. A.; El-Gendy, D. M.; Ahmed, N.; Banks, C. E.; Allam, N. K.; Biosens. Bioelectron. 2017, 15, 90.

15. Häkkinen, M.; Launiainen, T.; Vuori, E.; Ojanperä, I.; Forensic Sci. Int. 2012, 222, 327.

16. Armstrong, S. C.; Cozza, K. L.; Psychosomatics 2003, 44, 515.

17. Bjune, K.; Stubhaug, B. A.; Dodgson, M. S.; Breivik, H.; Acta Anaesthesiol. Scand. 1996, 40, 399.

18. Hau, V. S.; Huber, J. D.; Campos, C. R.; Davis, R. T; Davis, T. P.; Brain Res. 2004, 1018, 257.

19. World Anti-Doping Agency; Prohibited List; 2019. Available at https://www.wada-ama.org/sites/default/files/wada_2019_ english_prohibited_list.pdf accessed in September 2019.

20. World Anti-Doping Agency; Minimum Required Performance Levels for Detection and Identification of Non-Threshold Substances. Available at https://www.wada-ama.org/sites/ default/files/resources/files/td2018mrpl_v1_finaleng.pdf accessed in September 2019.

21. Mattia, C.; Ferrari, A.; Minerva Med. 2014, 105, 501.

22. Straube, C.; Derry, S.; Jackson, K. C.; Wiffen, Bell R. F.; Strassels, S.; Straube, S.; Cochrane Database Syst. Rev. 2014, CD006601.

23. Zhang, W. Y.; Po, A. L. W.; J. Clin. Pharm. Ther. 1996, 21, 261.

24. Anderson, M. S.; Silva, T. A.; Vicentini, F. C.; Fatibello-Filho, O.; Arabian J. Chem. 2017, DOI 10.1016/j.arabjc.2017.04.012.

25. Lamei, N.; Ezoddin, M.; Ardestani, M. S.; Abdi, K.; Anal. Bioanal. Chem. 2017, 409, 6113.

26. Lin, Z.; Li, J.; Zhang, X.; Qiu, M.; Huang, Z.; Rao. Y.; J. Chromatogr. B 2017, 1046, 177.

27. Ahmar, H.; Nejati-Yazdinejad, M.; Najafi, M.; Hasheminasab, K. S.; Chromatographia 2018, 81, 1063.

28. Meng, L.; Zhang, W.; Meng, P.; Zhu, B.; Zheng, K.; J. Chromatogr. B 2015, 989, 46.

29. Ebrahimzadeh, H.; Mirbabaei, F.; Asgharinezhad, A. A.; Shekari, N.; Mollazadeh, N.; J. Chromatogr. B 2014, 947, 75.
30. Karami, M.; Yamini, Y.; Abdossalami Asl, Y.; Rezazadeh, M.; J. Chromatogr. A 2017, 1527, 1.

31. Souza, I. D.; Hantao, L. W.; Queiroz, M. E. C.; Anal. Chim. Acta 2019, 1045, 108.

32. Kole, P. L.; Venkatesh, G.; Kotecha, J.; Sheshala, R.; Biomed. Chromatogr. 2011, 25, 199.

33. Goh, S. X. L.; Lee, H. K.; Anal. Chim. Acta 2018, 1019, 74.

34. Wang, R.; Li, W.; Chen, Z.; Anal. Chim. Acta 2018, 1018, 111.

35. Risticevic, S.; Lord, H.; Gorecki, T.; Arthur, C. L.; Pawliszyn, J.; Nat. Protoc. 2010, 5, 122.

36. Pena-Pereira, F.; Lavilla, I.; Bendicho, C.; TrAC, Trends Anal. Chem. 2010, 29, 617.

37. Pena-Pereira, F.; Lavilla, I.; Bendicho, C.; Spectrochim. Acta, Part B 2009, 64, 1.

38. Leong, M.-I.; Fuh, M.-R.; Huang, S.-D.; J. Chromatogr. A 2014, 1335, 2.

39. Seidi, S.; Rezazadeh, M.; Yamini, Y.; TrAC, Trends Anal. Chem. 2018, 108, 296.

40. Rezaee, M.; Yamini, Y.; Faraji, M.; J. Chromatogr. A 2010, 1217, 2342 .

41. Shishov, A.; Wieczorek, M.; Kościelniak, P.; Dudek-Adamska, D.; Telk, A.; Moskvin, L.; Bulatov, A.; Talanta 2018, 181, 359.

42. Sharifi, V.; Abbasi, A.; Nosrati, A.; J. Food Drug Anal. 2016, $24,264$.

43. Ghasemi, E.; J. Chromatogr. A 2012, 1251, 48.

44. Moinfar, S.; Khayatian, Gh.; Milani-Hosseini, M. R.; Talanta 2014, 129, 309.

45. Karimaei, M.; Sharafi, K.; Moradi, M.; Ghaffari, H. R.; Biglari, H.; Arfaeinia, H.; Fattahi, N.; Anal. Methods 2017, 9, 2865.

46 Ahmadi-Jouibari, T.; Pasdar, Y.; Pirsaheb, M.; Fattahi, N.; Anal. Methods 2016, 9, 980.

47. Moinfar, S.; Khayatian, Gh.; Microchem. J. 2017, 132, 293.

48. Moinfar, S.; Khayatian, Gh.; Jamil, L. A.; J. Iran. Chem. Soc. 2018, 15, 2511.

49. Akramipour, R.; Hemati, M.; Fattahi, N.; Pirsaheb, M.; AhmadiJouibari, T.; J. Pharm. Biomed. Anal. 2017, 140, 155.

50. Ahmadi-Jouibari, T.; Fattahi, N.; Mirzaei, N.; Sharafi, K.; Ghafari, H. R.; New J. Chem. 2017, 41, 11948.

51. Zahedi, P.; Hosseiny Davarani, S. S.; Moazami, H. R.; Nojavan, S.; J. Pharm. Biomed. Anal. 2016, 117, 485.

52. Yamini, Y.; Pourali, A.; Seidi, S.; Rezazadeh, M.; Anal. Methods 2014, 6, 5554.
Submitted: June 27, 2019

Published online: September 16, 2019 